\title{
The Impact of Viral and Host Elements on HIV Fitness and Disease Progression
}

\author{
Kenneth R. Henry, PhD, Jan Weber, PhD, \\ Miguel E. Quiñones-Mateu, PhD, and Eric J. Arts, PhD
}

\author{
Corresponding author \\ Eric J. Arts, PhD \\ Division of Infectious Diseases, BRB 1029, Case Western Reserve \\ University, 10900 Euclid Avenue, Cleveland, OH 44106, USA. \\ E-mail: eja3@po.cwru.edu
}

Current HIV/AIDS Reports 2007, 4:36-41

Current Medicine Group LLC ISSN 1548-3568

Copyright ( 2007 by Current Medicine Group LLC

Twenty-five years after the emergence of HIV onto the global scene, multiple advancements have been made in the understanding of HIV pathology. Thanks to the development of antiretroviral therapies, growing numbers of individuals with HIV infection experience slowed or halted acceleration to AIDS. Despite this, new HIV infections and AIDS-related morbidity and mortality are still common in the highly active antiretroviral therapy era. Recently, we and others have identified viral replicative fitness as a major determinant of HIV disease progression, which could have a major impact in the clinical setting. Therefore, in this review, we will discuss host and viral factors that affect viral fitness and its relationship on HIV pathogenesis.

\section{Introduction}

HIV is a rapidly mutating and adaptable pathogen resulting in an increase in viral genetic diversity and potentially an evolutionary edge. This high mutation rate $\left(3 \times 10^{-5}\right.$ substitutions/site/generation), recombinogenic properties, and large production of virions contribute highly to the pathogenesis of the virus [1-3]. Although its error-prone replication produces many nonviable virions, viable missense variants and recombinant viruses contribute to the HIV quasispecies [1,4,5]. In fact, the constant increase in the HIV population assists the adaptive nature of the virus, which positively affects HIV fitness and ultimately disease progression $[5,6 \bullet, 7]$. Contrarily, immune pressure can counteract high genetic diversity of HIV by continually eliminating a significant proportion of virus particles and infected cells [8-10]. For example, a strong immune response is associated with slow progression to AIDS, high CD4 cell counts, and low viral loads $[11,12]$. However, continual evolution during this selective pressure may also lead to immune escape, host adaptation, altered tissue/cell tropism, and a net increase in replicative fitness [13]. Thus, diversity of intrapatient HIV quasispecies is likely controlled by different viral and host factors. Regardless of the model, HIV quasispecies will evolve from the founder or infecting isolate and increase in heterogeneity during the course of disease [14,15]. Until recently, a direct relationship between increasing heterogeneity and fitness of an RNA virus has only been characterized in in vitro models $[6 \bullet, 16-18]$.

The progression of HIV infection to AIDS is unique for each individual, typically ranging from 3 to more than 14 years [19]. Although the range of progression varies, the molecular process does not deviate much. Immediately after transmission, the host enters the acute stage where viremia is high and the immune system is activated (Fig. 1). During this phase, the depletion of CD4+ T cells occurs rapidly in the gut and other mucosal sites [20-23]. This rapid depletion is not observed systemically and may be the result of coreceptor tropism and/or viral fitness. During the chronic stage, CD4+ T cells in the blood are gradually depleted and the mucosal CD4+ T cells never rebound (Fig. 1). This gradual decrease is often associated with the immune system counteracting the viral expansion and diversity. During AIDS, a dramatic increase is observed in viremia, corresponding to a rapid decline in systemic CD4+ T cells [23].

In 1996, CCR5 and CXCR4 were identified as two major coreceptors for HIV entry [24]. Based upon coreceptor usage, viruses are classified as CCR5-tropic (R5), CXCR4-tropic (X4), or dual-tropic (R5/X4) [25]. Interestingly, coreceptor usage may be an indication of HIV disease progression [25]. The R5 virus is in most cases responsible for the initial infection [25]. Not surprisingly, the majority of depleted cells in the mucosal areas are CD4+ CCR 5+ T cells which account for the bulk of T cells on the mucosal surface [20-22]. Additionally, CCR5 and CD4 can be found on the surface of dendritic cells (DCs), macrophages, and natural killer (NK) cells. Presumably, cellular landscaping may account for the bottleneck which eliminates the X4 viruses during sexual transmission [25]. However, X4 viruses are often associated with late stages of disease progression. 


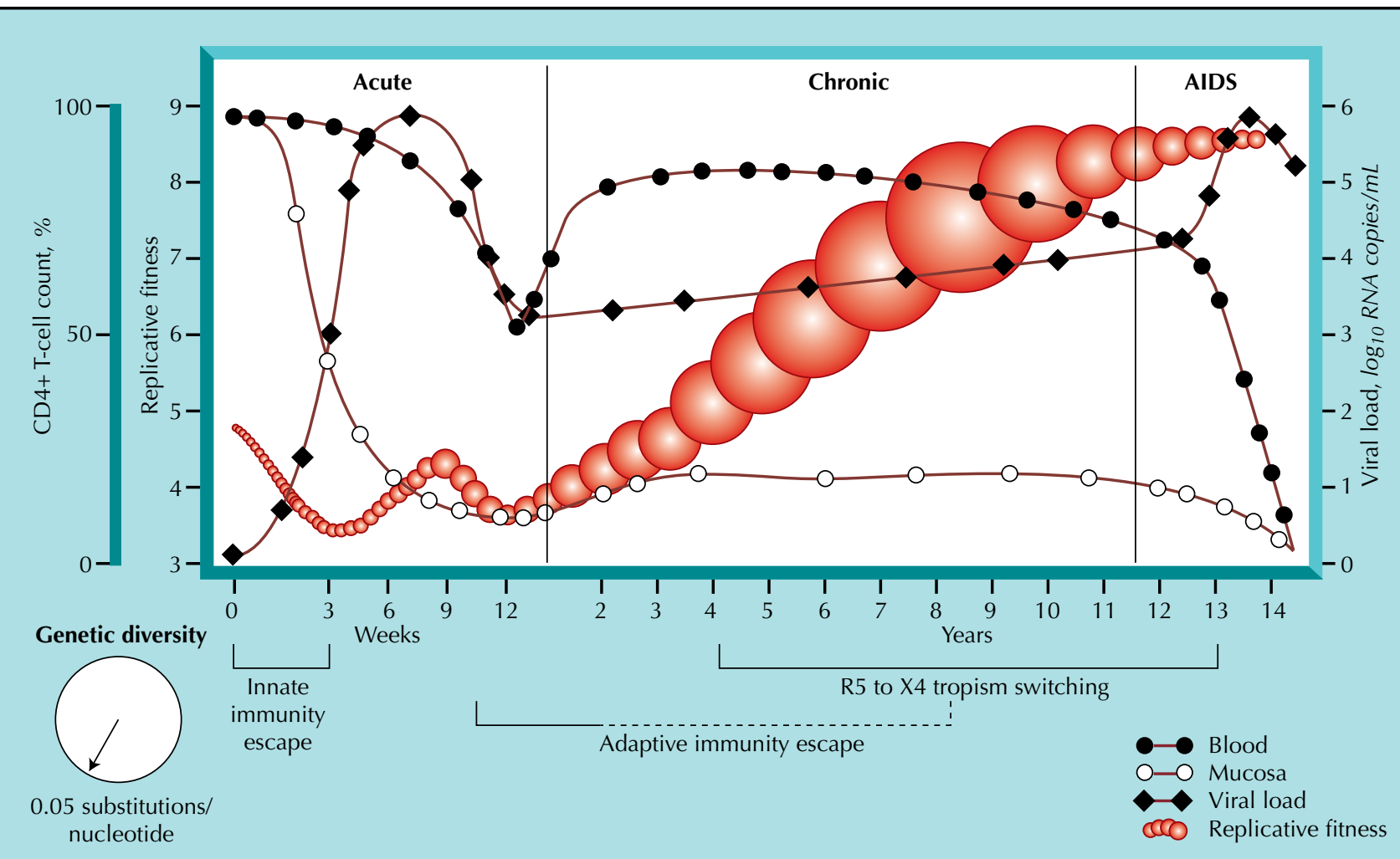

Figure 1. Fitness and disease progress model. Changes in replicative fitness and genetic diversity, CD4+ T-cell count in the blood and the mucosa, and viral loads are shown during the HIV disease progression. During acute phase, HIV encounters pressures from the innate immune response, resulting in a decline in replicative fitness. The virus then escapes innate immunity, and fitness, diversity, and viral load increase. Also, mucosal CD4+ T cells are rapidly depleted with the early weeks. Later in the acute stage, the adaptive immune system kicks in, and decreases are observed in viral load, fitness, and diversity. During the chronic phase, HIV adaptive immunity escape occurs, and replicative fitness increases. In the late chronic or AIDS stages, R5 and X4 viral tropism occurs in one half of the infected patients. All CD4+ T cells are depleted during the AIDS stage, and the viral load increases. Fitness remains high, but HIV diversity shrinks. Adapted from Shankarappa et al. [15].

HIV disease progression involves strong selection pressure which requires the virus to adapt to survive. During this adaptation, changes in viral fitness are often observed. In this context, HIV fitness is defined as the replicative capacity of the virus and its ability to survive within a host $[4,5]$. Due to the complex nature of the host species, several studies have explored the correlation of ex vivo fitness and disease progression $[6 \bullet, 7]$. Here we will focus on host and viral factors that affect HIV fitness and disease progression.

\section{Host Factors \\ CCR5 coreceptor}

As described above, HIV often goes through a fitness bottleneck during transmission between hosts, which reduces the viral population and general fitness $[4,26,27]$. HIV transmission predominately occurs at the mucosa by R 5 viruses due to the high population of CCR $5+\mathrm{CD} 4+$ cells [20-22]. Thus, mutations and polymorphisms in the CCR5 coreceptor may modulate viral transmission and fitness. The majority of subjects who were homozygous for a naturally occurring deletion of CCR 5 (ie, CCR5- $\Delta 32$ ) were found to be resistant to HIV-1 infection through sexual transmission [28]. Furthermore, infected individuals who are heterozygous for the CCR $5-\Delta 32$ alleles progressed at a slower rate toward AIDS than those lacking the deletion [28]. On the contrary, a known polymorphism in the promoter region of the gene (ie, allele P1) increases the expression of CCR5, resulting in an acceleration toward AIDS [29]. In the context of CCR5 P1 allele, these data suggest that there are more viral targets thus decreasing the stringency of the HIV-1 genetic bottleneck introduced by the host. This heightened HIV diversity allows "fitter" viruses to enter the new host which results in the observed acceleration of disease.

CCR5 and CD4 are found on mucosal innate immune cells such as macrophages, DCs, Langerhan cells (LC), NK cells, and NK T cells. These cells serve several functions in the protection of the host such as destroying foreign invaders, secreting cytokines, recognizing patterns, signaling cell proliferation, and activating the adaptive immune system. During sexual transmission of HIV, mucosal innate immune cells are the first direct and indirect line of defense; however, these cells are early targets of R5 viruses. The virus infects these cells or uses 
them for transport to lymphatic centers where it can infect CD4+ lymphocytes [30-32]. Infection of macrophages, DCs, LCs, and NK cells persists for weeks to months unlike the rapid destruction of CD4+ cells [30,31,33,34]. The fitness of viruses isolated from macrophages can also differ greatly from those isolated from CD4+ T cells [31].

\section{Chemokines}

Chemokines such as macrophage inflammatory proteins (MIP1 $\alpha$ and MIP1 $\beta$ ), regulated on activation normal T-cell expressed and secreted protein (RANTES), and stroma-derived factor (SDF- $\alpha 1 \mathrm{a}$ ) can inhibit HIV entry by two means: competing for coreceptor binding or downregulating the coreceptor on the surface of the cells [35-40]. Decreased expression of RANTES due to a polymorphism in the CCL5 gene (CCL5 In1.1c) accelerates HIV disease progression whereas the opposite effect was observed with RANTES upregulation [41]. More recently, it was shown that multiple copies of the CCL $3 L 1$ gene lead to higher circulating levels of CCL3L1 and slower disease progression [42•]. Moreover, in vitro studies indicate that treatment with chemokines such as MIP- $1 \alpha$, RANTES, and SDF- $1 \alpha$ blocks infection initially [39,41]. However, viral escape mutant can occur quickly (Fig. 1). This selective pressure potentially leads to viral escape through coreceptor tropism switching (R5 to X4 or vice versa), continued use of the same receptor in a different manner, or use of entirely different coreceptor (such as Bob/GPR15 or CXCR6) [43-47]. Karlsson et al. [48] showed that R5 viruses isolated later during disease progression are fitter than those isolated earlier in the presence of RANTES, making it less sensitive to the chemokine interference [48]. These data suggest that selective pressure from RANTES allow the virus to escape and increased fitness in presence of RANTES at physiological conditions. Thus, chemokines could have early effects on disease progression and fitness of the virus.

\section{Major histocompatibility complex class I and cytotoxic $T$ lymphocytes}

A decrease in viral fitness may also be due to an active and effective HIV-specific immune response and a continual selection of escape mutants. Three human leukocyte antigen (HLA) genes (HLA-A, HLA-B, HLA-C) encode the major histocompatibility complex (MHC) class I. MHC class I presents antigenic epitopes to cytotoxic T lymphocytes (CTLs). Polymorphic HLA alleles at these gene loci expand the breath of antigen presentation and can result in control of viremia and slowed disease progression in individuals with HIV infection by MHC class I interaction with HIV-specific CTLs [49-51]. Homozygosity at these loci limits antigen presentation which in turns limits epitopes recognized by CTLs and results in rapid disease progression in individuals with HIV infection [52].

Allen et al. [53] suggest that CTL selective escape is a major factor in HIV sequence diversity, although with evolutionary constraints. Even though they observed an increase in HIV diversity, a little more than one half of the new amino acid substitutions were associated with a CTL response; that is, $18 \%$ of CTL escape mutations were substitutions found in common subtype consensus sequences although these sequences had no or weak association with MHC expressed by the individual [53]. In nonhuman primates, various groups have shown that rapid viral escape from CTL selection pressure occurs within days. However, when the CTL escape virus was transmitted to a MHCmismatched host, the mutation reverted to the wild-type within weeks [54-56]. Additionally, selective pressure by HIV-specific CTL reduces HIV-1 diversity, selects for resistance mutations, and results in reductions in relative fitness [57,58]. However, in the case of CTL escape mutations, decreased fitness is short lived and rapidly compensated by secondary mutations [58]. These results strongly imply that viral escape from CTL selective pressure has a high fitness cost, and there are evolutionary constraints on the virus during the process of escape. CTL escape mutants become stable after a cycle of fitness loss and incremental gain (Fig. 1). Following CTL escape, the HIV-specific humoral response is possibly the second greatest threat to the HIV population. Many studies have shown that humoral response is less apt to control viremia than CTL response [59]. Escape from the humoral immunity is extremely rapid and continuous throughout disease progression [59].

\section{Viral Factors}

\section{The HIV envelope and tropism}

As described above, HIV infection in humans is generally established by R5 viral population during sexual activity. The initial establishment of infection by R 5 viruses is due to high expression of CCR5 on the mucosa $[20,21]$. Even after parenteral exposure, $\mathrm{R} 5$ viruses are predominant during early stages of disease progression [25]. The R5 virus phenotype usually persists throughout asymptomatic disease; however in approximately one half of late infections, the infecting HIV-1 isolates switch coreceptor tropism to $\mathrm{X} 4$ and utilize the CXCR4 receptor for entry [25]. This tropism switch from $\mathrm{R} 5$ to $\mathrm{X} 4$ often leads to rapid progression to AIDS. It has been thought this acceleration to AIDS is due to an increase in viral fitness; however X4 and $\mathrm{R} 5$ viruses can be equivalent in relative fitness [7]. On the other hand, the potential increase in X4 variant pathogenicity can be attributed to its ability to infect thymocytes at high levels (R5 viruses do not productively infect these cells) and/or to the fact that $\mathrm{X} 4$ viruses transfer much more efficiently between DCs to CD4+ than R5 variants [25,60]. In addition to dictating viral tropism, the HIV envelope seems to be major determinant of HIV fitness [39,61-63].

\section{Nef}

Evidence that other HIV genetic alterations could affect disease progression was clearly demonstrated in a few 
long-term nonprogressor patients who harbored HIV strains with nef deletions $[64,65]$. Interestingly, viruses with similar nef deletions were shown to be replication defective in peripheral blood mononuclear cell cultures [66]. Nef has been implicated in the downregulation of MHC class I, CD28, and CD4 resulting in viral evasion of the immune system [67]. Nef from nonpathogen simian immunodeficiency virus (SIV) infections in natural primate hosts mediates the downregulation of the T-cell receptor (TCR)/CD3 complex, which suppresses the susceptibility of infected $\mathrm{T}$ cells to activation and activation-induced cell death [67•]. However, HIV and a subset of SIV nef alleles failed to downregulate TCR/CD3 complex and to prevent cell death [67•]. Thus, the nef gene is clearly linked to viral fitness and disease progression.

\section{HIV recombinants}

Recombination is a common feature of retroviruses which serves as a mutagenic strategy for rapid evolution and adaptation. Recombination among different members of HIV quasispecies occurs in individuals infected with a single HIV strain. However, dual-infection and/or superinfection with additional HIV strains usually results in intrasubtype or intersubtype recombination $[13,68]$. As a consequence, HIV recombination can greatly increase genetic diversity and rapidly improve viral fitness. Often in the case of intersubtype recombinants, the recombinant form of two subtypes is relatively fitter than either parental virus $[68,69]$.

\section{Conclusions}

Although the impact of HIV fitness on disease progression in vivo is not thoroughly understood, strong correlations between HIV disease advancement and fitness have been established $[6 \bullet, 7,70]$. Studies on SIV pathogenesis showed that antigenic and cytopathic properties of the SIV strain predict fitness in the host [71,72]. Emerging SIV variants have increased replicative capacity over the SIV strain used in the initial infection. Additionally, infectious dose and viral fitness of the initial inoculum influences viral load during disease progression in SIV-infected macaques [73]. Decreased replicative fitness can be linked to slower disease progression, which could result in increased opportunity for transmission. The spread of such viruses poses practical challenges to future diagnostic tests and molecular epidemiologic studies. Thus, monitoring HIV fitness may prove to be important for clinical practice due to its strong correlation with HIV pathogenesis. Early detection of "fitter" recombinant or missense viral variants could possibly improve treatment to slow disease progression, ultimately providing clinicians with additional information and tools to combat the AIDS epidemic.

\section{References and Recommended Reading}

Papers of particular interest, published recently, have been highlighted as:

- Of importance

- Of major importance

1. Jung A, Maier R, Vartanian JP, et al.: Multiply infected spleen cells in HIV patients. Nature 2002, 418:144.

2. Mansky LM, Temin HM: Lower in vivo mutation rate of human immunodeficiency virus type 1 than that predicted from the fidelity of purified reverse transcriptase. I Virol 1995, 69:5087-5094.

3. Lal RB, Chakrabarti S, Yang C: Impact of genetic diversity of HIV-1 on diagnosis, antiretroviral therapy and vaccine development. Indian J Med Res 2005, 121:287-314.

4. Domingo E, Holland JJ: RNA virus mutations and fitness for survival. Annu Rev Microbiol 1997, 51:151-178.

5. Domingo E, Verdaguer N, Ochoa WF, et al.: Biochemical and structural studies with neutralizing antibodies raised against foot-and-mouth disease virus. Virus Res 1999, 62:169-175.

6. Troyer RM, Collins KR, Abraha A, et al.: Changes in human immunodeficiency virus type 1 fitness and genetic diversity during disease progression. J Virol 2005, 79:9006-9018.

This study establishes a correlation between HIV fitness and disease progression.

7. Quinones-Mateu ME, Ball SC, Marozsan AJ, et al.: A dual infection/competition assay shows a correlation between ex vivo human immunodeficiency virus type 1 fitness and disease progression. J Virol 2000, 74:9222-9233.

8. Lukashov VV, Goudsmit J: Founder virus population related to route of virus transmission: a determinant of intrahost human immunodeficiency virus type 1 evolution? J Virol 1997, 71:2023-2030.

9. Nowak MA, Anderson RM, McLean AR, et al.: Antigenic diversity thresholds and the development of AIDS. Science 1991, 254:963-969.

10. Nowak MA, May RM, Phillips RE, et al.: Antigenic oscillations and shifting immunodominance in HIV-1 infections. Nature 1995, 375:606-611.

11. Delwart EL, Pan H, Sheppard HW, et al.: Slower evolution of human immunodeficiency virus type 1 quasispecies during progression to AIDS. J Virol 1997, 71:7498-7508.

12. Wu H, Kuritzkes DR, McClernon DR, et al.: Characterization of viral dynamics in human immunodeficiency virus type 1-infected patients treated with combination antiretroviral therapy: relationships to host factors, cellular restoration, and virologic end points. J Infect Dis 1999, 179:799-807.

13. Quinones-Mateu ME, Gao Y, Ball SC, et al.: In vitro intersubtype recombinants of human immunodeficiency virus type 1: comparison to recent and circulating in vivo recombinant forms. J Virol 2002, 76:9600-9613.

14. Shankarappa R, Gupta P, Learn GH Jr, et al.: Evolution of human immunodeficiency virus type 1 envelope sequences in infected individuals with differing disease progression profiles. Virology 1998, 241:251-259.

15. Shankarappa R, Margolick JB, Gange SJ, et al.: Consistent viral evolutionary changes associated with the progression of human immunodeficiency virus type 1 infection. J Virol 1999, 73:10489-10502.

16. Clarke DK, Duarte EA, Elena SF, et al.: The red queen reigns in the kingdom of RNA viruses. Proc Natl Acad Sci U S A 1994, 91:4821-4824.

17. Escarmis C, Carrillo EC, Ferrer M, et al.: Rapid selection in modified BHK-21 cells of a foot-and-mouth disease virus variant showing alterations in cell tropism. J Virol 1998, 72:10171-10179. 
18. Novella IS, Duarte EA, Elena SF, et al.: Exponential increases of RNA virus fitness during large population transmissions. Proc Natl Acad Sci U S A 1995, 92:5841-5844.

19. Pantaleo G, Graziosi C, Fauci AS: The immunopathogenesis of human immunodeficiency virus infection. $N$ Engl J Med 1993, 328:327-335.

20. Brenchley JM, Schacker TW, Ruff LE, et al.: CD4+ T cell depletion during all stages of HIV disease occurs predominantly in the gastrointestinal tract. J Exp Med 2004, 200:749-759.

21. Guadalupe M, Reay E, Sankaran S, et al.: Severe CD4+ Tcell depletion in gut lymphoid tissue during primary human immunodeficiency virus type 1 infection and substantial delay in restoration following highly active antiretroviral therapy. J Virol 2003, 77:11708-11717.

22. Mehandru S, Poles MA, Tenner-Racz K, et al.: Primary HIV-1 infection is associated with preferential depletion of CD4+ T lymphocytes from effector sites in the gastrointestinal tract. J Exp Med 2004, 200:761-770.

23. Picker LJ, Watkins DI: HIV pathogenesis: the first cut is the deepest. Nat Immunol 2005, 6:430-432.

24. Pierson TC, Doms RW: HIV-1 entry and its inhibition. Curr Top Microbiol Immunol 2003, 281:1-27.

25. Poveda E, Briz V, Quinones-Mateu M, et al.: HIV tropism: diagnostic tools and implications for disease progression and treatment with entry inhibitors. AIDS 2006, 20:1359-1367.

26. Ho DD: HIV-1 dynamics in vivo. J Biol Regul Homeost Agents 1995, 9:76-77.

27. Lazaro E, Escarmis C, Perez-Mercader J, et al.: Resistance of virus to extinction on bottleneck passages: study of a decaying and fluctuating pattern of fitness loss. Proc Natl Acad Sci U S A 2003, 100:10830-10835.

28. Dean M, Carrington M, Winkler C, et al.: Genetic restriction of HIV-1 infection and progression to AIDS by a deletion allele of the CKR5 structural gene. Hemophilia Growth and Development Study, Multicenter AIDS Cohort Study, Multicenter Hemophilia Cohort Study, San Francisco City Cohort, ALIVE Study. Science 1996, 273:1856-1862.

29. Martin MP, Dean M, Smith MW, et al.: Genetic acceleration of AIDS progression by a promoter variant of CCR5. Science 1998, 282:1907-1911.

30. Fortis C, Poli G: Dendritic cells and natural killer cells in the pathogenesis of HIV infection. Immunol Res 2005, 33:1-21.

31. Goodenow MM, Rose SL, Tuttle DL, et al.: HIV-1 fitness and macrophages. J Leukoc Biol 2003, 74:657-666.

32. Turville $S$, Wilkinson J, Cameron P, et al.: The role of dendritic cell C-type lectin receptors in HIV pathogenesis. $J$ Leukoc Biol 2003, 74:710-718.

33. Valentin A, Pavlakis GN: Natural killer cells are persistently infected and resistant to direct killing by HIV-1. Anticancer Res 2003, 23:2071-2075.

34. Valentin A, Rosati M, Patenaude DJ, et al.: Persistent HIV-1 infection of natural killer cells in patients receiving highly active antiretroviral therapy. Proc Natl Acad Sci U S A 2002, 99:7015-7020.

35. Alkhatib G, Combadiere C, Broder CC, et al.: CC CKR5: a RANTES, MIP-1alpha, MIP-1beta receptor as a fusion cofactor for macrophage-tropic HIV-1. Science 1996, 272:1955-1958.

36. Amara A, Gall SL, Schwartz O, et al.: HIV coreceptor downregulation as antiviral principle: SDF-1alpha-dependent internalization of the chemokine receptor CXCR4 contributes to inhibition of HIV replication. J Exp Med 1997, 186:139-146.

37. Aramori I, Ferguson SS, Bieniasz PD, et al.: Molecular mechanism of desensitization of the chemokine receptor CCR-5: receptor signaling and internalization are dissociable from its role as an HIV-1 co-receptor. Embo J 1997, 16:4606-4616.

38. Arenzana-Seisdedos F, Virelizier JL, Rousset D, et al.: HIV blocked by chemokine antagonist. Nature 1996, 383:400.
39. Bleul CC, Farzan M, Choe H, et al.: The lymphocyte chemoattractant SDF-1 is a ligand for LESTR/fusin and blocks HIV-1 entry. Nature 1996, 382:829-833.

40. Mack M, Schlondorff D: Downmodulation and recycling of chemokine receptors. Methods Mol Biol 2000, 138:191-195.

41. Gallo RC, Garzino-Demo A, DeVico AL: HIV infection and pathogenesis: what about chemokines? J Clin Immunol 1999, 19:293-299.

42. Gonzalez E, Kulkarni H, Bolivar H, et al.: The influence of CCL3L1 gene-containing segmental duplications on HIV1/AIDS susceptibility. Science 2005, 307:1434-1440.

The study provides further evidence that host genetic alterations can impact HIV susceptibility particularly in the case of CCL3L1.

43. Cilliers T, Willey S, Sullivan WM, et al.: Use of alternate coreceptors on primary cells by two HIV-1 isolates. Virology 2005, 339:136-144.

44. Este JA, Cabrera C, Blanco J, et al.: Shift of clinical human immunodeficiency virus type 1 isolates from $\mathrm{X} 4$ to $\mathrm{R} 5$ and prevention of emergence of the syncytium-inducing phenotype by blockade of CXCR4. J Virol 1999, 73:5577-5585.

45. Mosier DE, Picchio GR, Gulizia RJ, et al.: Highly potent RANTES analogues either prevent CCR5-using human immunodeficiency virus type 1 infection in vivo or rapidly select for CXCR4-using variants. J Virol 1999, 73:3544-3550.

46. Schols D, Este JA, Cabrera C, et al.: T-cell-line-tropic human immunodeficiency virus type 1 that is made resistant to stromal cell-derived factor 1 alpha contains mutations in the envelope gp120 but does not show a switch in coreceptor use. J Virol 1998, 72:4032-4037.

47. Torre VS, Marozsan AJ, Albright JL, et al.: Variable sensitivity of CCR5-tropic human immunodeficiency virus type 1 isolates to inhibition by RANTES analogs. J Virol 2000, 74:4868-4876.

48. Karlsson I, Antonsson L, Shi Y, et al.: Coevolution of RANTES sensitivity and mode of CCR5 receptor use by human immunodeficiency virus type 1 of the $\mathbf{R} 5$ phenotype. J Virol 2004, 78:11807-11815.

49. Carrington M, Nelson GW, Martin MP, et al.: HLA and HIV-1: heterozygote advantage and $\mathrm{B} * 35-\mathrm{Cw}^{*} 04$ disadvantage. Science 1999, 283:1748-1752.

50. Gao X, Nelson GW, Karacki P, et al.: Effect of a single amino acid change in MHC class I molecules on the rate of progression to AIDS. N Engl J Med 2001, 344:1668-1675.

51. Kaslow RA, Carrington M, Apple R, et al.: Influence of combinations of human major histocompatibility complex genes on the course of HIV-1 infection. Nat Med 1996, 2:405-411.

52. Kamp W, Berk MB, Visser CJ, et al.: Mechanisms of HIV-1 to escape from the host immune surveillance. Eur J Clin Invest 2000, 30:740-746.

53. Allen TM, Altfeld M, Geer SC, et al.: Selective escape from CD8+ T-cell responses represents a major driving force of human immunodeficiency virus type 1 (HIV-1) sequence diversity and reveals constraints on HIV-1 evolution. J Virol 2005, 79:13239-13249.

54. Fernandez CS, Stratov I, De Rose R, et al.: Rapid viral escape at an immunodominant simian-human immunodeficiency virus cytotoxic T-lymphocyte epitope exacts a dramatic fitness cost. J Virol 2005, 79:5721-5731.

55. Friedrich TC, Dodds EJ, Yant LJ, et al.: Reversion of CTL escape-variant immunodeficiency viruses in vivo. Nat Med 2004, 10:275-281.

56. Friedrich TC, Frye CA, Yant LJ, et al.: Extraepitopic compensatory substitutions partially restore fitness to simian immunodeficiency virus variants that escape from an immunodominant cytotoxic-T-lymphocyte response. J Virol 2004, 78:2581-2585.

57. Martinez-Picado J, Prado JG, Fry EE, et al.: Fitness cost of escape mutations in p24 Gag in association with control of human immunodeficiency virus type 1. J Virol 2006, 80:3617-3623. 
58. Troyer R, Abraha A, Krizan R, et al.: The fitness cost of CTL escape: not a terrible hardship on HIV-1? Paper presented at the XVI International AIDS Conference.

Toronto, Canada; August 13-19, 2006.

59. Richman DD, Wrin T, Little SJ, et al.: Rapid evolution of the neutralizing antibody response to HIV type 1 infection. Proc Natl Acad Sci U S A 2003, 100:4144-4149.

60. Arien KK, Gali Y, El-Abdellati A, et al.: Replicative fitness of CCR5-using and CXCR4-using human immunodeficiency virus type 1 biological clones. Virology 2006, 347:65-74.

61. Ball SC, Abraha A, Collins KR, et al.: Comparing the ex vivo fitness of CCR5-tropic human immunodeficiency virus type 1 isolates of subtypes B and C. J Virol 2003, 77:1021-1038.

62. Marozsan AJ, Moore DM, Lobritz MA, et al.: Differences in the fitness of two diverse wild-type human immunodeficiency virus type 1 isolates are related to the efficiency of cell binding and entry. J Virol 2005, 79:7121-7134.

63. Rangel HR, Weber J, Chakraborty B, et al.: Role of the human immunodeficiency virus type 1 envelope gene in viral fitness. J Virol 2003, 77:9069-9073.

64. Deacon NJ, Tsykin A, Solomon A, et al.: Genomic structure of an attenuated quasi species of HIV-1 from a blood transfusion donor and recipients. Science 1995, 270:988-991.

65. Kirchhoff F, Greenough TC, Brettler DB, et al.: Brief report: absence of intact nef sequences in a long-term survivor with nonprogressive HIV-1 infection. N Engl J Med 1995, 332:228-232.

66. Daniel MD, Kirchhoff F, Czajak SC, et al.: Protective effects of a live attenuated SIV vaccine with a deletion in the nef gene. Science 1992, 258:1938-1941.
67.• Schindler M, Munch J, Kutsch O, et al.: Nef-mediated suppression of $T$ cell activation was lost in a lentiviral lineage that gave rise to HIV-1. Cell 2006, 125:1055-1067.

Demonstrates the significance of nef in the downregulation of TCR-CD3 which inhibits T-cell activation and ultimately prevents cell death.

68. Quinones-Mateu ME, Arts EJ: Recombination in human immunodeficiency virus type-1 (HIV-1): update and implications. AIDS Review 1999, 1:89-100.

69. Konings FA, Burda ST, Urbanski MM, et al.: Human immunodeficiency virus type 1 (HIV-1) circulating recombinant form 02_AG (CRF02_AG) has a higher in vitro replicative capacity than its parental subtypes A and G. J Med Virol 2006, 78:523-534.

70. Quinones-Mateu ME, Arts EJ: Virus fitness: concept, quantification, and application to HIV population dynamics. Curr Top Microbiol Immunol 2006, 299:83-140.

71. Kimata JT, Kuller L, Anderson DB, et al.: Emerging cytopathic and antigenic simian immunodeficiency virus variants influence AIDS progression. Nat Med 1999, 5:535-541.

72. Whetter LE, Ojukwu IC, Novembre FJ, et al.: Pathogenesis of simian immunodeficiency virus infection. J Gen Virol 1999, 80:1557-1568.

73. Holterman L, Niphuis H, Koornstra W, et al.: The rate of progression to AIDS is independent of virus dose in simian immunodeficiency virus-infected macaques. J Gen Virol 2000, 81:1719-1726. 